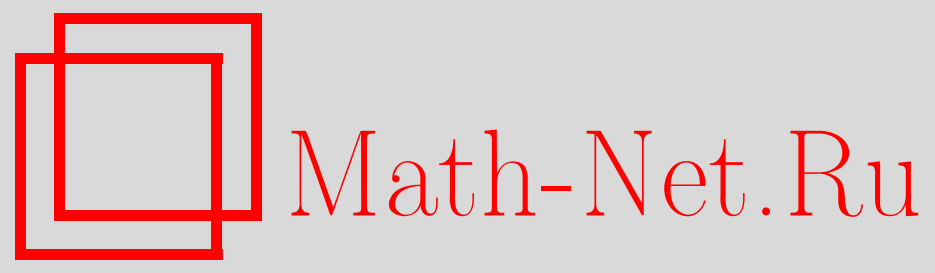

В. Д. Соловьев, Структура распределения информации в бесконечной последовательности, Дискрет. матем., 1996, том 8, выпуск 2, 97-107

DOI: https://doi.org/10.4213/dm524

Использование Общероссийского математического портала Math-Net.Ru подразумевает, что вы прочитали и согласны с пользовательским соглашением http: //www.mathnet.ru/rus/agreement

Параметры загрузки:

IP : 54.174 .149 .18

26 апреля 2023 г., 13:16:19 


\title{
Структура распределения информации в бесконечной последовательности
}

\author{
(c) 1996 г. В. Д. Соловьев
}

\begin{abstract}
Ставится задача изучения структуры распределения информации в бесконечных последовательностях. Для ее решения предложен подход, основанный на восстановлении последовательности по ее подпоследовательности. Для реализации әтого подхода вводится необходимый инструментарий, в частности, понятия жесткой и плотно упакованной последовательностей, характеризующие степень дублирования информации в последовательности. Рассматривается автоматная модель, в рамках которо доказано существование плотно упакованных и жестких последовательностей и установлен ряд их свойств, в частности, связь со сложностью предсказания очередного әлемента последовательности.
\end{abstract}

Продемонстрирована возможность применения полученных результатов к изучению структуры степеней автоматных преобразований, доказано, что любое конечное частично упорядоченное множество с наименьшим и наибольшим элементами изоморфно начальному сегменту структуры степеней автоматных преобразований последовательностей в алфавите $\{0,1\}$. В статье обсуждается отношение полученных результатов к теории информации и кодирования.

Работа выполнена при поддержке Российского фонда фундаментальных исследований, грант 93-011-16004.

Рассмотрим произвольную бесконечную последовательность символов некоторого конечного алфавита и поставим вопрос о характере распределения информации в этой последовательности. Например, в какой мере она дублирована, насколько равномерно распределена.

Примем следующий подход к изучению структуры распределения информации в бесконечной последовательности. Выбираем некоторую подпоследовательность и стираем информацию в этой подпоследовательности, скажем, заменяя все символы в этой подпоследовательности на 0. Затем пытаемся, используя оставшуюся часть исходной последовательности, восстановить стертую информацию. В зависимости от успешности восстановления можно судить об уникальности информации, содержащейся в подпоследовательности, степени дублирования информации в исходной последовательности.

Априорно можно ожидать существование последовательностей со следующими противоположными свойствами. Приведем неформальные определения таких последовательностей. 
Последовательность называется жесткой, если никакая ее собственная подпоследовательность не содержит полной информации, необходимой для восстановления всей исходной последовательности.

Последовательность называется плотно упакованной, если существуют сколь угодно редкие подпоследовательности, содержащие всю информацию исходной последовательности.

Для получения конкретных результатов необходимо уточнить, какие последовательности рассматриваются и какие средства используются для восстановления стертой информации. Настоящая статья посвящена автоматной модели.

В автоматной модели рассматриваются периодические подпоследовательности, а восстановленная последовательность получается на выходе некоторого конечного автомата при подаче на его вход частично стертой последовательности.

Доказано существование как жесткой, так и плотно упакованной последовательностей. Получена характеристика плотно упакованных последовательностей в терминах предсказания очередных символов последовательности. Введенные понятия могут быть применены к изучению структуры степеней автоматных преобразований бесконечных последовательностей: с использованием плотно упакованных последовательностей описаны конечные начальные сегменты степеней $(0,1)$-последовательностей.

1. Приведем необходимые определения.

Под конечным автоматом будет пониматься конечный инициальный автомат Мили $T=\left\langle X, S, Y, \delta, \lambda, s_{0}\right\rangle$ с начальным состоянием $s_{0} \in S$, функцией переходов $\delta: S \times X \rightarrow S$ и функцией выходов $\lambda: S \times X \rightarrow Y$. Если слово $U$ на входе автомата $T$ преобразуется в слово $W$, то пишем $T(U)=W$.

Конечный автомат может рассматриваться и как преобразователь бесконечных последовательностей. Если при подаче на вход автомата $T$ последовательности $A$ на выходе получается последовательность $B$ (может быть с конечной задержкой), то будем писать $B \leqslant A$ и говорить, что последовательность $B$ сводится к последовательности $A$ (автоматом $T$ ).

Для данной бесконечной последовательности $A$ множество последовательностей $\{B \mid B \leqslant A \& A \leqslant B\}$ называется степенью автоматных преобразований последовательности $A$ и обозначается $\operatorname{deg}(A)$. Сводимость последовательностей индуцирует частичный порядок и на множестве степеней.

Структура степеней автоматных преобразований, обозначаемая буквой $V$, была введена Рейна [1]. Он показал, что эта структура обладает наименьшим элементом, состоящим из периодических (с предпериодом) последовательностей и обозначаемым 0, а также атомами, то есть такими степенями $a$, что

$$
a>0 \& \neg \exists b(0<b<a)
$$

Для последовательности $A$ символ $A(i)$ обозначает $i$-й по счету әлемент последовательности $A$. Начинается последовательность $A$ с әлемента $A(0)$.

Определение 1. Пусть $A$ - произвольная бесконечная последовательность в алфавите $X$ и $\alpha$ - периодическая $(0,1)$-последовательность, т.е. периодическая 
последовательность в алфавите $\{0,1\}$. Последовательность $B$, для которой

$$
B(i)= \begin{cases}A(i), & \text { если } \alpha(i)=1, \\ 0, & \text { если } \alpha(i)=0,\end{cases}
$$

называется $\alpha$-прореженной из $A$. Последовательность называется $\alpha$-прореженной, если она $\alpha$-прорежена из $A$ для некоторой $A$.

Легко видеть, что для любой периодической $(0,1)$-последовательности $\alpha$ существует конечный автомат, преобразующий произвольную последовательность $A$ в $\alpha$-прореженную из $A$ последовательность. Другими словами, если $B$ - $\alpha$-прореженная из $A$ последовательность, то $\operatorname{deg}(B) \leqslant \operatorname{deg}(A)$.

Если $\alpha$ - периодическая $(0,1)$-последовательность, то $\bar{\alpha}$ будет обозначать последовательность, получающуюся из $\alpha$ заменой 0 на 1 и наоборот; $|\alpha|$ означает долю единиц в периодической последовательности $\alpha$.

Определение 2. Последовательность $A$ называется жесткой, если для любой периодической $(0,1)$-последовательности $\alpha$, содержащей бесконечное число 0 , $\alpha$-прореженная из $A$ последовательность имеет строго меньшую степень, чем $A$.

Определение 3. Последовательность $A$ называется плотно упакованной, если существует совокупность периодических $(0,1)$-последовательностей $\alpha_{1}, \alpha_{2}, \ldots$, обладающая следующими свойствами:

(1) $\forall i\left(\left|\alpha_{i}\right| \leqslant 2^{-i}\right)$,

(2) $\forall i$ ( $\alpha_{i}$-прореженная из $A$ последовательность имеет ту же степень, что и $\left.A\right)$.

Определение 4. Пусть для некоторой последовательности $\alpha$ последовательность $A$ является $\alpha$-прореженной, а последовательность $B$ является $\bar{\alpha}$-прореженной. Тогда последовательность $C$, для которой

$$
C(i)= \begin{cases}B(i), & \text { если } \alpha(i)=0, \\ A(i), & \text { если } \alpha(i)=1,\end{cases}
$$

называется нормальным сочленением (относительно $\alpha$ ) последовательностей $A$ и $B$.

Легко видеть, что степень последовательности $C$ является наименьшей верхней гранью степеней последовательностей $A$ и $B$.

Теорема 1. Любой атом структуры степеней автоматных преобразований состоит только из плотно упакованных последовательностей.

Доказательство. Пусть $A-$ произвольная последовательность из степени, являющейся атомом структуры $V$ стеденей автоматных преобразований. Построим совокупность периодических $(0,1)$-последовательностей $\left\{\alpha_{i}\right\}_{i=1}^{\infty}$, обладающую требуемыми в определении 3 свойствами. Параллельно с $\left\{\alpha_{i}\right\}_{i=1}^{\infty}$ строим совокупность $\left\{A_{i}\right\}_{i=1}^{\infty}$ бесконечных последовательностей. 
Положим

$$
\alpha_{1}^{\prime}(i)= \begin{cases}1, & \text { если } i \text { четно, } \\ 0, & \text { если } i \text { нечетно. }\end{cases}
$$

Либо $\alpha_{1}^{\prime}$-прореженная из $A$, либо $\overline{\alpha_{1}^{\prime}}$-прореженная из $A$ последовательность имеет ту же степень, что и $A$, так как в противном случае, если бы обе эти последовательности были периодическими (то есть имели строго меньшую степень, чем последовательность $A$ ), то и $A$, будучи нормальным сочленением относительно $\alpha_{1}^{\prime}$ әтих последовательностей, была бы периодической, вопреки предположению о том, что последовательность $A$ принадлежит атому. Допустим, что $\alpha_{1}^{\prime}$-прореженная из $A$ последовательность имеет ту же степень, что и $A$. Тогда положим $\alpha_{1}=\alpha_{1}^{\prime}$ и в качестве $A_{1}$ выбираем $\alpha_{1}^{\prime}$-прореженную из $A$ последовательность, в противном случае положим $\alpha_{1}=\overline{\alpha_{1}^{\prime}}$ и в качестве $A_{1}$ выбираем $\overline{\alpha_{1}^{\prime}}$-прореженную из $A$ последовательность.

Пусть $\alpha_{i}$ и $A_{i}$ уже определены и $A_{i}$ имеет ту же степень, что и $A$. Пусть $k_{1}, k_{2}, \ldots$ - номера единиц последовательности $\alpha_{i}$. Положим

$$
\alpha_{i+1}^{\prime}(j)= \begin{cases}0, & \text { если } \alpha_{i}(j)=0, \\ 0, & \text { если } j=k_{2 s+1}, s \geqslant 0, \\ 1, & \text { если } j=k_{2 s}, s>0 .\end{cases}
$$

Либо $\alpha_{i+1}^{\prime}$-прореженная из $A_{i}$, либо $\overline{\alpha_{i+1}}$-прореженная из $A_{i}$ последовательность имеет ту же степень, что и $A_{i}$ (а значит и $A$ ), так как $A_{i}$ является нормальным сочленением относительно $\alpha_{i+1}^{\prime}$ этих двух последовательностей. Если ту же степень, что и $A_{i}$ имеет $\alpha_{i+1}^{\prime}$-прореженная из $A_{i}$ последовательность, то полагаем $\alpha_{i+1}=\alpha_{i+1}^{\prime}$ и в качестве $A_{i+1}$ выбираем $\alpha_{i+1}^{\prime}$-прореженную из $A_{i}$ последовательность, в противном случае полагаем $\alpha_{i+1}=\overline{\alpha_{i+1}^{\prime}}$ и в качестве $A_{i+1}$ выбираем $\overline{\alpha_{i+1}^{\prime}}$-прореженную из $A_{i}$ последовательность.

Из построения ясно, что выполняются свойства 1,2 из определения 3 и, таким образом, последовательность $A$ плотно упакована. Теорема доказана.

Интуитивно ясно, что в случайной последовательности никакая ее регулярным образом выделенная часть не может быть восстановлена по оставшейся части случайной последовательности. И таким образом, естественно ожидать, что случайные последовательности, определяемые как, например, в формализации Мартин-Лефа, будут жесткими. Перед доказательством этого утверждения приведем определение случайной последовательности.

В пространстве $\Phi(0,1)$-последовательностей стандартным образом определяется мера $p$ на основе равенства

$$
p\left(\left\{A \mid A(0)=a_{0} \& \ldots \& A(n-1)=a_{n-1}\right\}\right)=2^{-n}
$$

для всех $a_{0}, \ldots, a_{n-1}$. Множество слов $K$ называется секвенциальным, если оно содержит вместе с каждым своим словом и все слова, содержащие данное слово в качестве начальной части. Для множества слов $K$ символ $[K]$ означает следующее множество бесконечных последовательностей: $\{B \mid$ некоторая начальная часть последовательности $B$ содержится в $K\}$.

Определение 5 ([2]). Тестом навывается эффективная последовательность $\left\{K_{i}\right\}_{i=1}^{\infty}$ рекурсивно перечислимых множеств слов в алфавите $\{0,1\}$ такая, что 
(1) $K_{1} \supseteq K_{2} \supseteq \ldots$,

(2) $\forall i\left(K_{i}\right.$ секвенциально),

(3) $\forall i\left(p\left(K_{i}\right) \leqslant 2^{-i}\right)$.

Случайной называется последовательность $A$ такая, что

$$
A \in \Phi-\bigcap_{i}\left[K_{i}\right]
$$

для любого теста $\left\{K_{i}\right\}_{i=1}^{\infty}$.

Предложение 1. Любая случайная последовательность является жесткой.

Доказательство. Пусть $A$ является случайной последовательностью в смысле определения 5 (такие, как известно [2], существуют) и $B-\alpha$-прореженная из $A$ последовательность для некоторой периодической $(0,1)$-последовательности $\alpha$, содержащей бесконечно много 0 . Докажем, что степень $B$ строго меньше степени $A$, что и означает, что $A$ жесткая.

Предположим противное, пусть $A \leqslant B$ посредством автомата $T$. Построим тест $\left\{K_{i}\right\}_{i=1}^{\infty}$. В этом тесте $K_{i}$ соответствует $i$-му 0 последовательности $\alpha$. Пусть в $(0,1)$-последовательности $\alpha$ цифра 0 встречается на местах с номерами $t_{1}, \ldots, t_{n}, \ldots$ Положим $K_{i}=\left\{Q \mid \exists U, W\left(U-\right.\right.$ слово длины $t_{i}$, имеющее 0 на местах с номерами $t_{1}, \ldots, t_{i} \& T(U)=W \& W$ - начальная часть слова $\left.\left.Q\right)\right\}$.

Выполнение условий 1-3 из определения 5 с $p\left(K_{i}\right)=2^{-i}$ для всех $i$ очевидно по построению последовательности $\left\{K_{i}\right\}_{i=1}^{\infty}$. Последовательность $\left\{K_{i}\right\}_{i=1}^{\infty}$ рекурсивно перечислима и, значит, $\left\{K_{i}\right\}_{i=1}^{\infty}$ - тест. Также очевидно, что

$$
\forall i\left(A \in\left[K_{i}\right]\right),
$$

то есть

$$
A \in \bigcap_{i}\left[K_{i}\right]
$$

и $A$ не случайна. Получено противоречие, что и завершает доказательство предложения.

2. В этом разделе мы охарактеризуем плотно упакованные последовательности в терминах предсказаний элементов последовательности по предшествующим.

Определение 6. Пусть $A$ произвольная бесконечная последовательность, $T$ конечный автомат и $T$ преобразует $A$ в последовательность $B$. Периодическую $(0,1)$-последовательность $\alpha_{T}$ назовем показателем предсказуемости последовательности $A$ автоматом $T$, если из $\alpha_{T}(i)=0$ следует, что $B(i)=A(i+1)$.

Теорема 2. Последовательность $А$ плотно упакована тогда и только тогда, когда для любого числа $k$ найдется конечный автомат $T$ тахой, что $\left|\alpha_{T}\right| \leqslant 2^{-k}$. 
Доказательство. Предположим, что $A$ плотно упакована, $\alpha_{k}$ периодическая $(0,1)$-последовательность такая, что $\left|\alpha_{k}\right| \leqslant 2^{-k}$, и $C-\alpha_{k}$-прореженная из $A$ последовательность, и следовательно, имеет ту же степень, что и $A$.

Пусть $m$ - период последовательности $\alpha_{k}$ и $0 \leqslant m_{1}<\ldots<m_{p}<m$ - номера единиц последовательности $\alpha_{k}$. По предположению $p / m \leqslant 2^{-k}$.

Пусть $T$ - конечный автомат, сводящий $A$ к $C$, и $s_{0}-$ его начальное состояние. Определим автомат $T^{\prime}$ следующим образом.

Входной и выходной алфавиты $T^{\prime}$ те же, что и у автомата $T$ (это алфавит, в котором задана последовательность $A$ ).

Состояниями автомата $T^{\prime}$ будут тройки $t=\left\langle s, s^{\prime}, i\right\rangle$, где $s, s^{\prime}-$ состояния автомата $T, i$ - целое число в интервале $[0, m-1]$.

Функции переходов $\delta_{T^{\prime}}$ и выходов $\lambda_{T^{\prime}}$ автомата $T^{\prime}$ следующие:

$$
\delta_{T^{\prime}}\left(\left\langle s, s^{\prime}, i\right\rangle, a\right)=\left\langle s^{\prime}, \delta_{T}\left(s^{\prime}, 0\right), i+1 \quad(\bmod m)\right\rangle,
$$

если $i \neq m_{1} \& \ldots \& i \neq m_{p}$ и

$$
\delta_{T^{\prime}}\left(\left\langle s, s^{\prime}, i\right\rangle, a\right)=\left\langle\delta_{T}(s, a), \delta_{T}\left(\delta_{T}(s, a), 0\right), i+1 \quad(\bmod m)\right\rangle
$$

в противном случае,

$$
\lambda_{T^{\prime}}\left(\left\langle s, s^{\prime}, i\right\rangle, a\right)=\lambda_{T}\left(s^{\prime}, 0\right),
$$

если $i \neq m_{1} \& \ldots \& i \neq m_{p}$, и

$$
\lambda_{T^{\prime}}\left(\left\langle s, s^{\prime}, i\right\rangle, a\right)=\lambda_{T}\left(\delta_{T}(s, a), 0\right)
$$

в противном случае.

Начальным состоянием автомата $T^{\prime}$ является состояние $t_{0}=\left\langle s_{0}, \delta_{T}\left(s_{0}, 0\right), 0\right\rangle$.

Пусть $\alpha_{T^{\prime}}$ - функция, являющаяся показателем предсказуемости последовательности $A$ автоматом $T^{\prime}$.

Из определения функции $\delta_{T^{\prime}}$ видно, что при подаче на вход автомата $T^{\prime}$ последовательности $A$ последовательность проходимых состояний

$$
t_{0}=\left\langle s_{0}, s_{0}^{\prime}, 0\right\rangle, t_{1}=\left\langle s_{1}, s_{1}^{\prime}, 1\right\rangle, \ldots
$$

будет обладать следующими свойствами: $s_{0}^{\prime}=\delta_{T}\left(s_{0}, 0\right)$ и $\forall i\left(i \neq m_{1}-1(\bmod m) \& \ldots \& i \neq m_{p}-1(\bmod m) \rightarrow s_{i}-\right.$ состояние, в которое перейдет автомат $T$ при подаче на вход первых $i-1$ символов последовательности $C$, а $s_{i}^{\prime}-$ состояние, в которое перейдет автомат $T$ при подаче на его вход $i$ символов последовательности $C$ ).

Но тогда, по определению функции $\lambda_{T^{\prime}}$, при $i \neq m_{1}-1(\bmod m), \ldots, i \neq m_{p}-1$ $(\bmod m)$ выход автомата $T^{\prime}$ при подаче на вход первых $i$ символов последовательности $A$ будет совпадать с выходом автомата $T$ при подаче на его вход первых $i+1$ символов последовательности $C$, т. е. с $(i+1)$-м символом последовательности $A$ (так как автомат $T$ по предположению преобразует $C$ в $A$ ).

Следовательно, $\alpha_{T^{\prime}}(i)$ может быть равна 1 только при

$$
i=m_{1}-1 \quad(\bmod m) \vee \ldots \vee i=m_{p}-1 \quad(\bmod m)
$$

и, значит, $\left|\alpha_{T^{\prime}}\right| \leqslant p / m \leqslant 2^{-k}$. 
Предположим обратное. Пусть последовательность $A$ такова, что для любого $k$ найдется автомат $T$ такой, что $\left|\alpha_{T}\right| \leqslant 2^{-k}$, где $\alpha_{T}-$ периодическая $(0,1)$-последовательность.

Если для заданного числа $k$ автомат $T$ таков, что $\alpha_{T}-$ периодическая $(0,1)$ последовательность с периодом $m$ и $\left|\alpha_{T}\right| \leqslant 2^{-k}$, то пусть $C-\alpha_{T}$-прореженная из $A$ последовательность и $0 \leqslant m_{1}<\ldots<m_{p}<m$ - номера единиц последовательности $\alpha_{T}$.

Определим автомат $T^{\prime}$ следующим образом. Состояниями автомата $T^{\prime}$ будут тройки $\langle u, s, i\rangle$, где $u$ - либо символ выходного алфавита $T$, либо символ $u_{0}$, не входящий в этот алфавит, $s$ - состояние автомата $T, i$ - число в интервале $[0, m-1]$.

Входной и выходной алфавиты автомата $T^{\prime}$ те же, что и у автомата $T$. Начальное состояние автомата $T^{\prime}$ равно $\left\langle u_{0}, s_{0}, 0\right\rangle$, где $s_{0}$ - начальное состояние автомата $T$.

Функция выходов определяется следующим образом:

$\lambda_{T^{\prime}}\left(\left\langle u_{0}, s_{0}, 0\right\rangle, a\right)=A_{0}$ - первый элемент последовательности $A$,

$\lambda_{T^{\prime}}(\langle u, s, i\rangle, a)=\lambda_{T}(s, u)$, если $i>0 \& i \neq m_{1}+1(\bmod m) \& \ldots \& i \neq m_{p}+1(\bmod m)$, $\lambda_{T^{\prime}}(\langle u, s, i\rangle, a)=a$, если $i=m_{1}+1(\bmod m) \vee \ldots \vee i=m_{p}+1(\bmod m)$.

Функция переходов определяется следующим образом:

$$
\delta_{T^{\prime}}(\langle u, s, i\rangle, a)=\left\langle\lambda_{T^{\prime}}(\langle u, s, i\rangle a), \delta_{T}(s, u), i+1 \quad(\bmod m)\right\rangle
$$

при $i>0$ и

$$
\delta_{T^{\prime}}\left(\left\langle u_{0}, s_{0}, 0\right\rangle, a\right)=\left\langle\lambda_{T^{\prime}}\left(u_{0}, s_{0}, 0\right), \delta_{T}\left(s_{0}, A(1)\right), 1\right\rangle .
$$

Методом индукции легко показывается, что при подаче на вход автомата $T^{\prime}$ последовательности $C$ на выходе получается последовательность $A$. Таким образом, $A$ сводится к $C$ и эти две последовательности имеют одинаковую степень, так как сводимость $C$ к $A$ следует из того, что $C-\alpha_{T}$-прореженная из $A$ последовательность.

Теорема доказана.

3. Покажем, как концепция плотно упакованных последовательностей может быть применена к изучению структуры степеней автоматных преобразований.

Определение 7. Степень $a$ называется неразложимой, если

$$
\neg \exists b, c(b<a \& c<a \& \forall d(b<d \& c<d \rightarrow a<d))
$$

(то есть степень $a$ не является наименьшей верхней гранью никаких отличных от нее двух степеней).

Обозначим $G$ множество степеней

$$
\{a \mid\{b \mid b \leqslant a\} \text { конечно }\} \text {. }
$$

Предложение 2. Если степень неразложима, то она содержсит $(0,1)$-последовательность. 
Доказательство. Пусть $A$ - последовательность в алфавите $\left\{a_{1}, \ldots, a_{n}\right\}, n>2$, содержащем наименьшее число символов среди всех последовательностей, принадлежащих неразложимой степени $a$. Пусть $k, k \geqslant 1$, - такое число, что $k^{2}<n \leqslant(k+1)^{2}$. Символы алфавита $\left\{a_{1}, \ldots, a_{n}\right\}$ можно рассматривать как коды пар (не обязательно всех) символов $\left\langle t_{1}, t_{2}\right\rangle$ из алфавита, содержащего не более $k+1<n$ символов.

Построим две новые последовательности $A_{1}$ и $A_{2}$ следующим образом: если символ $A(i)$ является кодом пары $\left\langle t_{1}, t_{2}\right\rangle$, то $A_{1}(i)=t_{1}$ и $A_{2}(i)=t_{2}$. Так как конечный код может быть представлен в памяти конечного автомата, степени последовательностей $A_{1}$ и $A_{2}$ меньше или равны степени последовательности $A$, которая является наименьшей верхней гранью этих степеней. В силу предположения о неразложимости степени последовательности $A$, либо $A_{1}$, либо $A_{2}$ принадлежат той же степени, что и $A$, но каждая из них в алфавите из меньшего числа символов, чем в алфавите последовательности $A$. Полученное противоречие доказывает предложение 2 .

Определение 8. Назовем степень автоматных преобразований плотно упакованной, если она содержит плотно упакованную последовательность.

Предложение 3. Любая плотно упакованная степень состоит только из плотно упакованных последовательностей.

Доказательство. Пусть $A$ - плотно упакованная последовательность, и $B-$ произвольная последовательность, входящая в ту же степень, что и $A$. Тогда существуют такие конечные автоматы $T_{1}$ и $T_{2}$, что $T_{1}(A)=B$ и $T_{2}(B)=A$ (конечной задержкой можно пренебречь). По теореме 2 для последовательности $A$ и любого числа $k$ найдется такой автомат $T$, что для последовательности $\alpha_{T}$ из определения 6 справедлива оценка $\left|\alpha_{T}\right| \leqslant 2^{-k}$.

Тогда автомат $T_{1} \circ T \circ T_{2}$, являющийся последовательной композицией автоматов $T_{2}, T$ и $T_{1}$, предсказывает элементы последовательности $B$ и, очевидно, $\left|\alpha_{T_{1} \circ T \circ T_{2}}\right| \leqslant 2^{-k}$. Таким образом, $B$ плотно упакована по теореме 2 . Предложение доказано.

Предложение 4. Любая неразлохимая степень автоматных преобразований является плотно упакованной.

Доказательство совершенно аналогично доказательству теоремы 1.

Теорема 3. Любая степень из $G$ содержит плотно упакованную $(0,1)$-последовательность.

Доказательство. Доказательство проводится индукцией по числу элементов множества $\{b \mid b \leqslant a\}$. Если степень $a$ неразложима (в том числе атом), то по предложению 2 она содержит некоторую $(0,1)$-последовательность $A$. Согласно предложению 4 эта последовательность является плотно упакованной.

Если степень $a$ разложима, то пусть $a$ является наименьшей верхней гранью степеней $b$ и $c$, строго меньших $a$. По индуктивному предположению $b$ и $c$ содержат плотно упакованные $(0,1)$-последовательности $B$ и $C$ соответственно. Пусть 
$\left\{\beta_{i}\right\}_{i=1}^{\infty}$ и $\left\{\gamma_{i}\right\}_{i=1}^{\infty}-$ совокупности периодических $(0,1)$-последовательностей, существующие, согласно определению 3 , для последовательностей $B$ и $C$ соответственно.

Рассмотрим периодическую последовательность $\beta_{1}$. Ее можно представить в виде

$$
\beta_{1}(x)=1 \leftrightarrow x \in\left\{k_{1}+n l \mid n \in \mathbf{N}\right\} \cup \ldots \cup\left\{k_{q}+n l \mid n \in \mathbf{N}\right\}, \quad k_{1}, \ldots, k_{q}<l .
$$

По предположению $\left|\beta_{1}\right| \leqslant 2^{-1}$, поэтому $q \leqslant l / 2$.

Последовательность $\gamma_{1}$ может быть представлена аналогичным образом. Без ограничения общности можно, выбрав некоторое общее кратное периодов последовательностей $\beta_{1}$ и $\gamma_{1}$, перейти к другим представлениям обеих последовательностей, но уже с одинаковым периодом.

Пусть $\gamma_{1}$ имеет вид

$\gamma_{1}(x)=1 \leftrightarrow x \in\left\{m_{1}+n l \mid n \in \mathbf{N}\right\} \cup \ldots \cup\left\{m_{t}+n l \mid n \in \mathbf{N}\right\}, \quad m_{1}, \ldots, m_{t}<l, \quad t \leqslant l / 2$

и $h_{1}, \ldots, h_{l-q}$ - все элементы множества $\left\{[0, l-1]-\left\{k_{1}, \ldots, k_{q}\right\}\right\}$. Пусть перестановка $\varepsilon:[0, l-1] \rightarrow[0, l-1]$ такова, что $\varepsilon\left(m_{1}\right)=h_{1}, \ldots, \varepsilon\left(m_{t}\right)=h_{t}$. Такая перестановка найдется, так как $t \leqslant l / 2 \leqslant l-q$.

Определим последовательности $\gamma_{1}^{\prime}$ и $C^{\prime}$ следующим образом: для $x=i+n l$

$$
\begin{aligned}
& C^{\prime}(x)=C\left(\varepsilon^{-1}(i)+n l\right), \\
& \gamma_{1}^{\prime}(x)=\gamma_{1}\left(\varepsilon^{-1}(i)+n l\right) .
\end{aligned}
$$

Конечность перестановки $\varepsilon$ позволяет реализовать ее конечным автоматом, и тем самым последовательность $C^{\prime}$ имеет ту же степень, что и $C$, и также является плотно упакованной. Кроме того

$$
\forall x\left(\beta_{1}(x)=1 \rightarrow \gamma_{1}^{\prime}(x)=0\right) .
$$

Отсюда следует, что существует нормальное сочленение относительно $\beta_{1}$ последовательностей $\beta_{1}$-прореженной из $B$ и $\gamma_{1}$-прореженной из $C^{\prime}$. Обозначим эту последовательность $A$. Очевидно, что $A$ является $(0,1)$-последовательностью степени $a$. Покажем, что $A$ плотно упакована.

Определим совокупность периодических $(0,1)$-последовательностей $\alpha_{1}, \alpha_{2}, \ldots$ следующим образом.

Для всех $j$ и для $x=i+n l$ положим

$$
\gamma_{j+1}^{\prime}(x)=\gamma_{j+1}\left(\varepsilon^{-1}(i)+n l\right),
$$

и пусть

$$
\alpha_{j}(x)=1 \leftrightarrow \beta_{j+1}(x)=1 \vee \gamma_{j+1}^{\prime}(x)=1
$$

Тогда

$$
\left|\alpha_{j}\right| \leqslant\left|\beta_{j+1}\right|+\left|\gamma_{j+1}^{\prime}\right| \leqslant 2^{-(j+1)}+2^{-(j+1)}=2^{-j}
$$

и $\alpha_{j}$-прореженная из $A$ последовательность является нормальным сочленением (относительно $\beta_{1}$ ) последовательностей, являющихся $\beta_{j+1}$-прореженной из $B$ и $\gamma_{j+1}^{\prime}$-прореженной из $C^{\prime}$. Так как степени последних двух последовательностей есть $b$ и $c$, степень $\alpha_{j}$-прореженной из $A$ последовательности есть $a$.

Согласно определению $3 A$ плотно упакована и теорема 3 доказана. 
Следствие 1. Любая степень из $G$ состоит только из плотно упахованных последовательностей.

Доказательство следует непосредственно из предложения 3 и теоремы 3.

В теории алгоритмов общим подходом к изучению различных структур степеней является описание начальных сегментов этих структур. Для структуры $V$ наиболее сильный результат получен С. С. Марченковым [3]. Согласно ему любое конечное частично упорядоченное множество с наименьшим и наибольшим элементами является начальным сегментом $V$.

Обозначим через $V_{2}$ структуру степеней $(0,1)$-последовательностей, определяемую аналогично $V$. Доказательство результата Марченкова существенным образом использует последовательности в алфавитах из более чем двух символов и, таким образом, не дает информации о начальных сегментах $V_{2}$.

Так как все конечные начальные сегменты содержатся в множестве степеней $G$, из теоремы 3 непосредственно вытекает следующий результат.

Следствие 2. Любое конечное частично упорядоченное множество с наименьшим и наибольшим әлементами является начальным сегментом $V_{2}$.

Таким образом, в данной работе показано существование бесконечных последовательностей с существенно различными структурами распределения информации в них. В последовательностях, названных жесткими, полностью отсутствует дублирование информации, любая часть жесткой последовательности несет уникальную информацию. В противоположность этому, плотно упакованные последовательности являются как бы бесконечными матрешками: сколь угодно малые части этих последовательностей содержат полную информацию исходных последовательностей. То есть здесь имеет место специфическим образом организованное бесконечное дублирование информации.

Аналог теоремы 2 для конечных слов хорошо известен в теории информации и кодирования [4]: чем точнее можно предсказывать символы текста, тем в большей степени он может быть сжат.

Подход, развиваемый в данной работе, отличается от классической теории кодирования следующими двумя главными аспектами:

(1) мы рассматриваем кодирование бесконечных последовательностей символов, в то время как в теории кодирования изучается кодирование конечных текстов,

(2) мы допускаем возможность массированных ошибок, например, может быть уничтожено $99,99 \%$ символов, в то время как в теории кодирования ошибки считаются редким явлением.

Суммируя, можно сказать, что развиваемый подход является предельным по отношению к теории кодирования. В некотором смысле он относится к теории кодирования так же, как Блюмовская теория сложности вычислений [6] к традиционной теории сложности.

Хотя результаты получены лишь в рамках автоматной модели, но, вероятно, обнаруженные эффекты носят устойчивый характер и могут быть получены и для других моделей при использовании для восстановления информации конечных преобразователей Гинсбурга [5] (ими являются кодеры и декодеры) или произвольных алгоритмов. 


\section{Список литературы}

1. Рейна Г. Степени автоматных преобразований. Киберн. сборних (1977) 14, 95-106.

2. Martin-Löf $P$. The definition of random sequences. Information and Control (1966) 9, 61-66.

3. Марченков С. С. Конечные начальные сегменты верхней полурешетки конечноавтоматных степеней. Дискретная математика (1989) 1, №3, 96-103.

4. Хэмминг Р. В. Теория кодирования и теория информачии. Радио и связь, Москва, 1983.

5. Ginsburg S. Examples of abstract machines. IEEE Trans. Electron. Computers (1962) 11, 132-135.

6. Блюм М. Машинно-независимая теория сложности рекурсивных функций. Пробл. матем. логики (1970).

Статья поступила 29.03.94. 